\title{
The role of personality in financial decisions and financial crises
}

\author{
Thomas Noe \\ Balliol College/SBS \\ Nir Vulkan \\ Woster College/SBS
}

$15 / 11 / 2017$

\begin{abstract}
In this chapter, we consider the role of personality traits in shaping the behavior of financial actors. We consider the promise of using personality to predict financial behavior. Personality is perhaps the ultimate "exogenous" predictive variable - stable throughout the lifetime of individuals and substantially heritable. At the same time, personality traits have demonstrated predictive power for agent actions. However, there are a number of inherent obstacles to incorporating personality into the standard economic model of financial decision making. We suggest some approaches to overcoming these obstacles.
\end{abstract}

Personality traits such as being "aggressive" are actively frequently listed as job qualifications in the finance industry (Endlich, 2000). Traders are commonly believed to have a "Wall street personality." One of the causes attributed to the last financial crisis was the aggressive risk taking of traders and bankers. Some of this risk-taking can no doubt be linked with compensation structures. However, it seems reasonable to consider whether the personalties of financial agents also played a role in the crisis. 
Because preventing the next crisis is even more important than understanding the last, if personality is an important determinant of the behavior of actors in the financial sector, the economics profession has an even more important task-provide policy makers and firms with tools they need to assess the effects of personality on aggregate economic outcomes. This requires building financial models that aggregate the effects of personality on individual actors and map these effects into economic models. In short, economist will need develop economic models that incorporate personality.

In the paper, we show that, although the evidence produced thus far is limited, it all points in one direction: personality has a significant independent effect on the decisions of economic actors. Thus, economic modeling should incorporate personality variables. However, personality research has been primarily developed with in social psychology. The social psychology perspective on decision making is fundamentally different from the economic and financial economics perspective. Thus, incorporation of personality into economics will be challenging. We lay out the issues involved and offer some tentative suggestions for surmounting the obstacles to developing "personality aware" models of financial behavior.

In the social-psychology literature, personality traits are defined as dispositional attributes of agents. Attributes that reflect the inherent traits of agents rather than their response to transitory stimuli from the 
agents' environment. So for example, everyone sometimes acts aggressively but everyone does not have an aggressive disposition.

As we discuss in detail below, personality traits are stable over time, measurable with well established instruments, and appear to be substantially heritable. In short, they represent a nearly perfect sort of exogenous variation between agents. In this respect, personality traits contrast sharply with the preference traits such as risk aversion, typically posited in economic models. Measurements of preference traits such as risk aversion are unstable and context dependent (Reynaud and Couture, 2012). In fact, frequently subject risk preferences cannot be measured at all because subject responses to survey instruments are frequently inconsistent.

Moreover, social-psychology research has established that these traits have considerable explanatory power for predicting behavior in general. Thus, it is not surprising they firms, recruiting agencies, and hiring consultants, make extensive use of personality measurement when making employment decisions. For example, ghSMART, is a consulting firm that advises many leading corporations, venture capital firms, and hedge funds on the suitability of candidates for top executive positions. The personalities of Job candidates referred to ghSMART are assessed through four-hour structured interview that results in a twenty to forty page assessment (Kaplan and Sorensen, 2016). The fact that firm are willing to pay for this sort of very expensive personality assessment, 
seems to indicate that they believe that personality has significant predictive power for future executive performance.

Despite this academic evidence from social psychology and the wholesale adoption of personality trait measurement in the finance industry, financial economics research has devoted little attention to the effect of personality traits on financial decision making. In the discussion below we explain this apparent paradox. First, in Section 1 we develop the definition of personality trait employed in social psychology and present some of the evidence for personality's effect on behavior. In Section 2 we consider in detail research on the effects of personality in economic and financial contexts. In Section 3 we consider possible approaches to fitting the personality model into economic models of decision making. Finally in Section 4 we consider these approaches in light of the evidence produced thus far on the effects of personality and suggest some possible approaches while, tentatively, ruling out some others.

\section{Personality}

Allport:1937,Allport:1961 defines personality as the dynamic organization of characteristics that creates a personâ€ $€^{\mathrm{TM}_{\mathrm{S}}}$ cognitions, motivation and behavior. Over the years, the study of personality psychology and individual differences encompassed many theoretical approaches. Arguably the most important approach is the trait 
(disposition) approach. Trait approaches assume that personality traits differ across individuals, but are stable within an individual (during adulthood) and over time(McCrae and Costa, 2003) and that these traits shape the personâ $€^{\mathrm{TM}_{\mathrm{S}}}$ behavior. ${ }^{1}$

The Five-Factor personality (FFM) Model (Costa and McCrae, 1992; Goldberg, 1993) (also known as the "Big 5" model) is the prominent trait-disposition theory of personality. According to this model, there are five major personality dimensions (or domains): Neuroticism, Extroversion, Conscientiousness, Agreeableness and Openness to Experience. Each of these dimensions is further composed of several different facets. The Big-5 model is empirically based, and the five factors as well as their facets have been derived using factor analysis. ${ }^{2}$ Psychological research accumulated a great deal of evidence regarding traits and specific behaviors. Given our focus on risk-taking behavior by financial agents, the most relevant traits are those associated with risk taking. Risk taking is most associated with one of the Big Five Traits-Neuroticism. Neuroticism, also defined as low emotional stability (Goldberg, 1993) is characterized by a tendency to experience negative affectivity and psychological distress. Neurotic individuals are

${ }^{1}$ Examples can be found in Barrick/Mount:1991, Hurtz/Donovan:2000, Hogan/Holland:2003, Mount:1994, Porpat:2009, Roberts/Kuncel/Shiner:2007 and ; but see Morgeson:2007 for a different perspective.

${ }^{2}$ In fact, most of the criticism of the Big 5 model revolves around the fact that the model is data driven rather than theory driven. See, for example, the comprehensive review by Block:2010. 
"ineffective in their attempts to cope with stress and are prone to engage in irrational thought" ( Bettencourt et al., 2006, p. 754). They are more likely to experience anxiety, anger, guilt and depression, and interpret ordinary situations as threatening (Matthews et al., 1998). The facets of neuroticism include anxiety, angry hostility, depression, self-consciousness, and impulsiveness. The facet of Neuroticism most closely connected with risk-taking is aggressiveness (angry hostility). ${ }^{3}$ Aggressiveness is intended to increase social dominance, and cause pain or harm to others (Ferguson and Beaver, 2009) and is associated with behaviors such as risk taking (Lerner and Keltner, 2001). ${ }^{4}$

Bettencourt:2006 present a meta-analytic review of personality and aggressive behavior. They conclude that personality should be included as a central variable in models of aggressive behavior. Marshall/Brown:2006 demonstrate that people who are higher on the aggressiveness trait are more reactive to provocation, resulting in more aggressive behavior. In a more related study, Lauriola/Levin:2001 demonstrate that individuals high in Neuroticism engage in less risky decisions in the gains domain, but more risk taking in the domain of losses.

${ }^{3}$ As opposed to antagonistic hostility that is associated with the (low) Agreeableness domain. We define aggressiveness as angry hostility rather than antagonistic hostility.

${ }^{4}$ We use the term anxiousness and aggressiveness to refer to the traits, while anxiety and aggression refer to states or behaviors. 


\section{Personality's effect on economic and financial}

\section{decisions}

Little research has been performed on the effect of personality traits on economic and financial behavior. However, even this limited body of research has documented strong associations between personality and economic behavior. Mcinish:1982 measured the personality traits of sophisticated investors using a Rotter scale measuring "locus-of control", the degree to which the investors believe that their own (internal) actions affect their life outcomes versus outside (external) forces beyond their control. He found that high scores for an external locus of control were associated with higher portfolio risk measured using the CAPM beta.

In an experimental asset market composed of subject students,vanWitteloostuijnEtal:2007 documented a strong relationship between personality variables and trading behavior. Subjects who scored

high on impatience and urgency exhibited higher-trading frequencies, lower price sensitivity, and earned lower arbitrage profits.

JohnsonEtAl:2009 experimentally implement a trust game in which, given trustworthy behavior by the trustee (agent being trusted), the trustor's (agent trusting) payoff is highest from trust and trustworthy behavior is a strictly dominant strategy for the trustee. The unique Nash equilibrium solution for this game is for the trustor to trust and the 
trustee to be trustworthy. However, the their experiment, a significant number of trustor's did not trust. JohnsonEtAl:2009 found this failure to trust even when trust is rational, was strongly associated with a personality facet, alienation.

RustichiniEtAl:2012 document the statistical relationship between personality traits and a number of life outcomes related to risk taking — smoking, body mass index, credit score, job attachment, and truck accidents. They find that the explanatory power of personality variables is a least as great as the explanatory power of standard economic variables (e.g., risk aversion) and that, in some cases, personality variables have more than double the explanatory power of economic variables.

Kugler/Neeman/Vulkan:2015 investigated anxiousness and aggressiveness in an experiment implementing two-player symmetric entry game, where each player can guarantee a certain payoff by staying out. The certain payoff is less than the payoff a player receives she is the only player to enter but a higher than the payoff if both players enter. Because the choice of an optimal strategy depends on the playerâ $€^{\mathrm{TM}_{S}}$ beliefs regarding the behavior of the other players, this experimental design permits identification not only of the effects of personality but also the effects agents' conjectures about the personalities of other agents. Kugler/Neeman/Vulkan:2015 found that [i.] 1. aggressive players entered significantly more often than non-aggressive players, 2 . 
anxious players entered significantly less often than non-anxious players, 3. players were less likely to enter when matched with aggressive players and more likely to enter when matched with anxious players. Thus, the behavior of subjects was not only significantly affected by their own personality traits but all with their beliefs about the personality traits of other players.

Kaplan/Sorensen:2016 examine personality data collected by major consulting firm, ghSmart, that specializes in personality evaluations for senior executive candidates. They find that the personality traits of CEO and non-CEO candidates differ significantly. Moreover, non-CEO candidates with high scores in "CEO" personality traits were more likely to eventually receive CEO appointments than non-CEO candidates with lower scores.

In a recent paper by the authors (Noe and Vulkan, 2016), we performed an experiment on seasoned financial professional. In the experiment, the participants received signals of the value of possible alternative investment to a market tracking portfolio. The signal were parameterized so that, under a standard Bayesian evaluation, the alternative investment was both risker and on average less profitable than investing in the tracking portfolio. We found that, in an individual decision context, personality factors had no explanatory power for investment decisions. However, in a group context, the personality trait aggressiveness had a large and economically significant positive effect 
on the probability of investing in the alternative asset. Moreover, in the group setting, standard economic variables had no significant impact on group decision making and aggressiveness was only weakly and negatively correlated with risk tolerance. In short, for individual decisions, standard economic variables, notably risk aversion, predicted behavior fairly well and personality variables played no role. In group decisions, economic variables played no role a personality variable, aggressiveness, had significant predictive power.

\section{Personality and financial economics paradigm}

The psychological literature focuses on individual behavior and decisions and, as such, is not directly applicable to the strategic situations (games) frequently encountered by financial decision makers. At the same time, it is not concerned with aggregation, determining the aggregate effects of individual choices on economic outcomes. Thus, in order for apply the insights from this literature into predictive theories of the effect of personality on economic behavior, it is necessary to incorporate them into a framework that does consider strategic interactions of economic agents and their aggregate effects-the financial economic paradigm.

In order to understand the issues associated with incorporating personality into the standard financial economics paradigm, it is necessary to review the essential aspects of this paradigm. We 
concentrate initially on the expected utility framework and later explain how cognitive biases and frequently be interpreted as distortions of either the probability weights or payoff function of the expected utility model.

Financial economics borrows its basic paradigm of human action from economics. In the economics paradigm, agents act to maximize their welfare conditioned on a given preferences over outcomes. In general this perspective permits preferences to be defined over choice sets that encompass the goods the agent personally consumes, the consumption pattern of other agents, public goods, etc.(e.g., Fehr and Schmidt, 1999). Moreover, a wide variety of preference structures have been analyzed, including even rather exotic preference structures (e.g., Fishburn, 1990). However, the vast majority of financial economics research, and almost all research aimed at developing testable structural models of the pricing and demand for financial assets, has worked with a "fragment" of this theory and assumed that agents have preferences determined solely by their own random payoffs and that these preferences can be represented by a utility function which maps random prospects into numbers, with higher numbers representing more preferred prospects. Because this framework is borrowed from economics we will refer to it as the "economic framework" even though it is not representative of all economics research on agent choice. 
To understand this framework, consider a financial decision in which the utility of an agent depends on a random prospect. To minimize the technical complexity of the analysis assume that the random prospect's realized payoff assumes one of $x_{i}$ possible values. A utility function maps random prospects, $X$, into numbers. If the utility function represents the preferences of a financial decision maker faithfully, then the number assigned to the prospect $X$ will be higher than the number assigned to $Y$ if and only if the decision maker prefers $X$ to $Y$. The financial decision maker's preferences are described by the expected utility model if for all random prospects, utility, $U$, is determined by

$$
U(X)=\sum_{i}^{n} u\left(x_{i}\right) p_{i}
$$

where $u$ is a utility-of-wealth function that maps the realized payoff of the prospect, $x_{i}$ into a number and $\pi_{i}$ is the probability that the decision maker assigns to $X=x_{i}$.

Thus, the utility of prospects is determined by preferences, measured by the utility of wealth function, $u$ and beliefs, measured by the decision-makers probability distribution over realizations of the random prospect, $p$. This probability distribution is based on a fixed prior probability but might be updated by information. If it is updated, updating is governed by Bayes rule. 
Behavioral theories of decision making can frequently be interpreted as injecting "distortions" into preferences or belief structures defined by the expected utility paradigm. For example under, under the most influential behavioral model of choice, prospect theory (Kahneman and Tversky, 1979), decision-maker utility, which we represent by $V$, is given by

$$
V(X)=\sum_{i}^{n} v\left(x_{i}\right) \pi\left(p_{i}\right),
$$

where $v$ is a valuation function for realized payoffs that is convex below a reference point, which represents the decision makers status-quo wealth, and concave above the reference point. $\pi$ is a probability weighting function that overweights small probabilities and underweight large probabilities. Although a decision maker whose utility is given by a prospect theory has preferences inconsistent with the axioms that imply expected utility maximization, formally, the prospect theory is model is quite similar to the expected utility model: Under both models, the decision maker has a payoff function representing preferences over realized outcomes, $u$ in the expected utility framework and $v$ in the prospect theory framework. Under both, the decision maker has beliefs that assign weights to these payoffs, the outcomes' probability, $p$, under expected utility and the weighted probability $\pi(p)$ under prospect theory. Under both the utility of the agent is a weighted sum of the payoffs. 
Of course, it is easy to posit structures of beliefs and payoffs under which the belief-weighted sum of payoffs determines the utility of decision makers. It is much harder to justify these strictures. Expected utility theory is justified by an axiomatic normative framework: a set of axioms is assumed to characterize rational choice over prospects. These axioms imply the expected utility representation.

Behavioral theories are justified by appeals to evidence from cognitive psychology. Cognitive psychology is ideally positioned to provide such evidence as its focus is on how agents form beliefs and preferences and how these beliefs and preference influence action. Thus, the output of cognitive psychology research is easy to input into justifications for behavioral models of decision making; behavioral biases modify preferences and/or beliefs, and these modified preferences and beliefs determine agent actions. For this reason, it is not surprising that the behavioral biases identified by cognitive psychology have been readily adopted to produce economic models which incorporate loss aversion (Tversky and Kahneman, 1991), overconfidence (Scheinkman and Xiong, 2003), guilt (Battigalli and Dufwenberg, 2007) and many other constructs from cognitive psychology.

Personality however falls in the domain of social psychology, and social psychology is not focused on preferences and beliefs. Rather, psychological research on personality focuses on the effect of a dispositional attributes of decision makers, personality traits, on agent 
actions. Thus, as framed by the social psychology literature, personality factors are not an input into the model of preferences and beliefs. Rather the social psychology framework "short-circuits" the economic model by positing a direct link between personality and the actions of decision makers. This difference in the conceptualization of the determinants of behavior between these two frameworks is illustrated in Figure Error! Reference source not found..

(a) Economic framework

(b) Social psychology framework.

Figure 1:

Thus, incorporating the effects of personality on economic decisions is a much more challenging task than incorporating cognitive bases into the economic model. Two approaches to incorporation are fairly apparent: justify a causal relation between personality and one or both determinants of decisions in the economic framework- preferences and beliefs_-or justify a "dual-self model" where agents normally make decisions based on a utility function maximization of the type considered by rational choice and behavioral economics but in some 
cases "personality takes over" and personality traits lead directly to decisions that are not rationalizable though agent utility functions.

The first approach to incorporation, which we term the integration approach, treats personality traits as inputs to formation of preference and/or beliefs. In contrast to the situation with cognitive psychology, integration does not simply require applying the results of the psychological literature to the economic variables. As discussed above there has been little research linking personality directly to beliefs and preferences. Thus, empirical research is required to document the nature of such links.

In the integration framework, personality has causal effect on beliefs and preferences. Beliefs and preferences then fix the behavior of decision makers. The causal structure of the integration approach is depicted in Figure 2.

\section{[Sorry. Ignored \begin } \{ \text { tikzpicture\} ... } \end\{tikzpicture\}] }}

Figure 2: Integration framework. Personalities effects are mediated through preferences and beliefs.

Although significant empirical evidence will be required to justify the sort of causal effects postulated by an integration approach, a priori 
there are many plausible justifications for causal relations between personality and preferences/beliefs. For example, aggressiveness has been linked to down-side risk taking. This evidence might be rationalized in the integration framework as resulting either from aggressiveness inducing utility-of-wealth functions that exhibit increasing absolute or relative risk aversion or by positing that aggressiveness leads to probability weighting functions that underweight the probability of losses. Note that the first alternative has personality acting on a rational-choice expected utility and the second on a behavioral objective function based on prospect theory. Thus, in principle, there is no logical impediment even to integrating personality into a non-behavioral rational-choice paradigm of decision making.

Many other causal links are also plausible. Conscientiousness might increase risk aversion. Aggressiveness, in competitive situations, might lead to agent utility functions that weighed more heavily on the rank of the agents payoff relative to other agents rather than the absolute magnitude of the payoff. Alternatively, aggressiveness might lead to overconfidence and thus an overweighting of the probabilities for upside payoffs.

The problem with the integration approach is not so much the lack of plausible hypotheses for causal relations but rather the lack of evidence to distinguish these hypotheses. Separating the effects of beliefs and 
preferences is a general and fairly intractable problem of identification in economics research.

Justification of the integration approach introduces an additional source of complexity: not only do the effects of beliefs and preferences have to be identified from observations of actions that are determined by both, but, in order to identify the causal mechanism thorough which personality acts, also th effect of personality must be "localized" to beliefs or preferences by the experimental design, i.e, by experiment structured so that personality will effect either beliefs or preferences but not both. For example, belief effects could be controlled through experiments involving complete information games. Such designs would fix beliefs and thus restrict personality to affecting preferences. These sorts of controls will require careful controlled experiments which are typically only possible in laboratory setting.

The advantage of the integration approach is that it incorporates personality into a unified theory of financial decision making. In fact, in a formal theoretical sense, under the integration approach, the standard model would not change at all. Economics research typically takes preferences and beliefs as exogenous inputs from which, using maximization, either under a rational-choice or behavioral objective function, predictions about behavior are generated. Whether or not these "inputs" derive from personality or some other source not relevant to predicting their effect on behavior conditioned on their effect on beliefs 
and preferences. Of course, when economic models are applied, identification of preferences and beliefs is required to derive determinant predictions of behavior. Personality, if it explains a significant variation in preference and beliefs, would thus greatly facilitate the application of economic models to real-world problems.

In contrast, the second possible approach to incorporating personality into economic analysis is to use it to bound the scope of application for the economic framework. This approach is consistent with research positing that economic agents have multiple "selves" and that which self makes a particular economic decision depends on the decision's context (Peleg and Yaari, 1973; Strotz, 1955). Using this idea, the social psychology model and the economic model might share control agent behavior. Instead of a unified theory of decision making this approach, which we term the dual-self approach, postulates two selves for economic agents a "calculating self" that makes utility maximizing choices using some consistent but perhaps behavioral utility function and a "dispositional self" that acts without calculation on the basis of personality traits. In some contexts, the dispositional self determines agent behavior, in others the rational self. The advantage of this approach is that it would not require integrating social psychology model of disposition and action into the economic model of utility maximization. The causal model which would underlie a dual-self formulation is presented in Figure 3. 


\section{[Sorry. Ignored \begin } \{ \text { tikzpicture\} ... } \end\{tikzpicture\}] }}

Figure 3: Dual-selfframework. Depending on the context, the dispositional or rational self controls behavior.

The advantage of dual-self model is that it does not require integrating personality into the economic model. Instead preferences and beliefs of the calculating self could be treated as exogenous and decisions produced by these beliefs and preference can be deduced from the standard economic calculus of optimization. Decisions produced by the dispositional self could be modeled simply as they are in social psychology, as primitive determinants of behavior.

However. this approach as a number of drawback which seem to outweigh this benefit. One obvious problem with the approach is determining when the calculating self as opposed to the dispositional self is controlling behavior. On this question, we have little insight, perhaps even less than we do for the potential effects of personality on preferences and beliefs. In addition, the dual-self approach would likely lead to models that predicted extreme shifts in agent behavior in response to small context changes. In a context that almost triggers the dispositional self, and agent behavior might be completely consistent with the axioms of expected utility. A small shift in the context that then 
triggered the dispositional self would result in large changes in behavior because personality traits, which do not affect the agents calculating self, would now completely determine the agent's dispositional-self behavior.

Finally, some fundamental results in financial economics impose extremely weak demands on trader rationality. For example, contingent claim pricing models that derive the relation between the price of an asset and the price of claims written on the asset (e.g., an option on the asset) are founded on arbitrage principles-deviations from the predicted price relationship between the asset and the contingent claim permit trading strategies that sometime generate gains over a fixed and finite time horizon, never generate losses, and require no commitment of capital. Although it is conceivable that some sort of triggering event might lead a shift in control of trader behavior the dispositional self and thus lead to, say, a bubble in asset prices, it is hard to imagine that the triggering event would also lead to a "bubble" in the relative price of assets and their contingent claims. No one has ever even advanced the idea that such relative price bubbles occur. Thus, to fit reality, a dual self model would need to condition trigger events not only on psychological variables but also on economic characteristics of the trading environment. Thus, the social psychology framework would still in some sense have to be integrated with the economic framework at the 
level of triggering events. Overall, the dual-self approach appears to be a less promising approach to incorporation than the integration approach.

\section{Tentative conclusions}

From our discussion one can conclude that (a) personality variables have significant predictive power for predicting economic behavior sometimes far more than the variables typically employed by economists and (b) these variables, being drawn from a different paradigm for human action, are difficult to integrate into the economic framework for decision making, (c) integration is required if personality is going to be used in testable structural models of aggregate economic behavior.

Thus far, progress has been limited on (b). However, research does provide some guidance about how integration might be accomplished. Most of guidance, unfortunately, is of an exclusionary variety-casting doubt on simple routes to integration rather than identifying the best route.

Perhaps the simplest route to integration is to integrate personality into the classical expected utility framework. In this framework, beliefs are fixed by prior probabilities and Bayesian updating. Preferences are determine solely by monetary rewards from random prospects. Thus, personality could affect decisions only thorough changing risk preferences. However, our work and the work of others suggest that personality variables are only weakly correlated with standard measures 
of risk attitudes even when they have significant effects on behavior (Rustichini et al., 2012; Noe and Vulkan, 2016). Thus, at least based on current evidence, it appears that the standard expected utility framework circumscribes the channels through which personality can act too much to permit the integration of personality into economic modeling.

Another somewhat more complex but nevertheless attractive approach would be to integrate personality into the belief formation process of agents whose decision calculus is affected by cognitive biases. However, our experimental results show that the effect of personality on decisions depends on social context of the decisions. We found that personality had strong effect in group decision contexts and an insignificant effect in individual decision contexts. One would not expect that an agent's cognitive biases would vary based on the social context. JohnsonEtAl:2009 implement a game in which probabilities are specified by the experimenter and where all choices for the agents are so stark that the solution of the game is the same for regardless of whether the agents are expected utility maximizers or maximizers of any standard behavioral utility function. Yet personality had a significant effect on players actions. When choices are invariant to belief distortion, personality's effect on beliefs should not affect outcomes. Thus, the evidence presented in JohnsonEtAl:2009 also seems inconsistent with personality only affecting beliefs. 
Thus, although integration might require incorporating the effect of personality on cognitive biases, it will almost certainly require integration of personality into valuation functions. This will require incorporating into the model of financial decision making more factors than simple monetary payoffs.

On obvious candidate for incorporation is suggested by an obvious difference between decisions in group and individual contexts, which our work suggests has significant effects on the power of personality to shape behavior. When making decisions in a group context we care not only about the monetary rewards generated by the decision but also about our status, whether we "came out on top" or at least did not come out on the bottom. Recently, there has been a great deal of research in economics on status-based preferences and risk-taking to attain status based rewards (Ray and Robson, 2012; Fang and Noe, 2016). Thus we conjecture that a promising avenue for future research is measuring the effect of personality variables, particularly variables such as aggressiveness, which seem by its nature to involve status concerns, on the strength of agents' status-based preferences.

Of course, there is very little evidence thus far on the contextual dependence of personality effect on decision making. Hence, there may well be other more promising routes for augmenting the classical utility-of-wealth or behavioral payoff fiction to incorporate arguments that are sensitive to personality. At this point, the jury is still out. 
Personality's effects on economic decision making are an elusive prey but one that will be quite rewarding to capture. The gains from capture will accrue not only to economic science but also to society at large because economic models informed by personality will be better able to capture warning signs for looming finance crises and thus provide policy makers the tools they require to avert them. 


\section{References}

[Allport]Allport1961Allport:1961 Allport, Gordon W, Pattern and growth in personality, Holt, Reinhart \& Winston, 1961.

[Allport]Allport1937Allport:1937 Allport, Gordon Willard, Personality, Holt New York, 1937.

[Barrick and Mount]Barrick and Mount1991Barrick/Mount:1991 Barrick, Murray R and Michael K Mount, "The big five personality dimensions and job performance: a meta-analysis," Personnel psychology, 1991, 44 (1), 1-26.

[Battigalli and Dufwenberg]Battigalli and Dufwenberg2007Battigalli/Dufwenberg:2007_Battigalli, Pierpaolo and Martin Dufwenberg, "Guilt in games," The American Economic Review, 2007, pp. 170-176.

[Bettencourt et al.]Bettencourt, Talley, Benjamin and Valentine2006Bettencourt:2006 Bettencourt, B, Amelia Talley, Arlin James Benjamin, and Jeffrey Valentine, "Personality and aggressive behavior under provoking and neutral conditions: a meta-analytic review," Psychological Bulletin, 2006, 132 (5), 751.

[Block]Block2010Block:2010 Block, Jack, “The five-factor framing of personality and beyond: Some ruminations," Psychological Inquiry, 2010, 21 (1), 2-25. 
[Costa and McCrae]Costa and McCrae1992Costa/McCrae:1992 Costa, Paul T and Robert R McCrae, "Four ways five factors are basic," Personality and Individual Differences, 1992, 13 (6), 653-665.

[Endlich]Endlich2000Endlich:2000 Endlich, Lisa, Goldman Sachs: The Culture of Success, Simon and Schuster, 2000.

[Fang and Noe]Fang and Noe2016Fang/Noe:2016 Fang, Dawei and Thomas H. Noe, "Skewing the odds: Taking risks for rank-based rewards," Working paper, Saïd Business School 2016.

[Fehr and Schmidt]Fehr and Schmidt1999Fehr/Schmidt:1999 Fehr, Ernst and Klaus M Schmidt, "A theory of fairness, competition, and cooperation," Quarterly Journal of Economics, 1999, 114 (3), $817-868$.

[Ferguson and Beaver]Ferguson and Beaver2009Ferguson/Beaver:2009 Ferguson, Christopher J and Kevin M Beaver, "Natural born killers: The genetic origins of extreme violence," Aggression and Violent Behavior, 2009, 14 (5), 286-294.

[Fishburn]Fishburn1990Fishburn:1990 Fishburn, Peter C, “Additive non-transitive preferences," Economics Letters, 1990, 34 (4), $317-321$.

[Goldberg]Goldberg1993Goldberg:1993 Goldberg, Lewis R, "The structure of phenotypic personality traits," American Psychologist, 1993, 48 (1), 26. 
[Hogan and Holland]Hogan and Holland2003Hogan/Holland:2003 Hogan, Joyce and Brent Holland, "Using theory to evaluate personality and job-performance relations: a socioanalytic perspective," Journal of Applied Psychology, 2003, 88 (1), 100.

[Hurtz and Donovan]Hurtz and Donovan2000Hurtz/Donovan:2000 Hurtz, Gregory M and John J Donovan, "Personality and job performance: the Big Five revisited," Journal of applied psychology, 2000, 85 (6), 869.

[Johnson et al.]Johnson, Rustichini and MacDonald2009JohnsonEtAl:2009 Johnson, Melissa K, Aldo Rustichini, and Angus W MacDonald, "Suspicious personality predicts behavior on a social decision-making task," Personality and Individual Differences, 2009, 47 (1), 30-35.

[Kahneman and Tversky]Kahneman and Tversky1979Kahneman/Tversky:1979 Kahneman, Daniel and Amos Tversky, "Prospect theory: An analysis of decision under risk," Econometrica, 1979, 47, 263-291.

[Kaplan and Sorensen]Kaplan and Sorensen2016Kaplan/Sorensen:2016 Kaplan, Steven and Martin Sorensen, “Are CEOs different? Characteristics of top managers," 2016. Working paper, Harvard Business School.

[Kugler et al.]Kugler, Neeman and Vulkan2014Kugler/Neeman/Vulkan:2015 Kugler, Tamar, Zvika 
Neeman, and Nir Vulkan, "Personality traits and strategic behavior: Anxiousness and aggressiveness in entry games," Journal of Economic Psychology, 2014, 42, 136-147.

[Lauriola and Levin]Lauriola and Levin2001Lauriola/Levin:2001 Lauriola, Marco and Irwin P Levin, "Personality traits and risky decision-making in a controlled experimental task: An exploratory study," Personality and Individual Differences, 2001, 31 (2), 215226.

[Lerner and Keltner]Lerner and Keltner2001Lerner/Keltner:2001 Lerner, Jennifer S and Dacher Keltner, "Fear, anger, and risk," Journal of Personality and Social Psychology, 2001, 81 (1), 146.

[Marshall and Brown]Marshall and Brown2006Marshall/Brown:2006 Marshall, Margaret A and Jonathon D Brown, "Trait aggressiveness and situational provocation: A test of the traits as situational sensitivities (TASS) model," Personality and Social Psychology Bulletin, 2006, 32 (8), 1100-1113.

[Matthews et al.]Matthews, Saklofske, Costa Jr, Deary and Zeidner1998MatthewsEtAl:1998 Matthews, Gerald, Donald H Saklofske, Paul T Costa Jr, Ian J Deary, and Moshe Zeidner, "Dimensional models of personality: A framework for systematic clinical assessment," European Journal of Psychological Assessment, 1998, 14 (1), 36. 
[McCrae and Costa]McCrae and Costa2003Mccrae/Costa:2003 McCrae, Robert R and Paul T Costa, Personality in Adulthood: A Five-Factor Theory Perspective, Guilford Press, 2003.

[McInish]McInish1982Mcinish:1982 McInish, Thomas H, "Individual investors and risk-taking," Journal of Economic Psychology, 1982, $2(2), 125-136$.

[Morgeson et al.]Morgeson, Campion, Dipboye, Hollenbeck, Murphy and Schmitt2007Morgeson:2007 Morgeson, Frederick P, Michael A Campion, Robert L Dipboye, John R Hollenbeck, Kevin Murphy, and Neal Schmitt, “Are we getting fooled again? Coming to terms with limitations in the use of personality tests for personnel selection,” Personnel Psychology, 2007, 60 (4), 10291049.

[Mount et al.]Mount, Barrick and Strauss1994Mount:1994 Mount, Michael K, Murray R Barrick, and J Perkins Strauss, "Validity of observer ratings of the big five personality factors," Journal of Applied Psychology, 1994, 79 (2), 272.

[Noe and Vulkan]Noe and Vulkan2016Noe/Vulkan:2016 Noe, Thomas and Nir Vulkan, "Naked aggression," Working paper, Saïd Business School, Oxford 2016.

[Peleg and Yaari]Peleg and Yaari1973Peleg/Yaari:1973 Peleg, Bezalel and Menahem E. Yaari, "On the Existence of a Consistent Course 
of Action when Tastes are Changing," The Review of Economic Studies, 1973, 40 (3), pp. 391-401.

[Poropat]Poropat2009Porpat:2009 Poropat, Arthur E, “A meta-analysis of the five-factor model of personality and academic performance," Psychological bulletin, 2009, 135 (2), 322.

[Ray and Robson]Ray and Robson2012Ray/Robson:2012 Ray, Debraj and Arthur Robson, "Status, intertemporal choice, and risk-taking," Econometrica, 2012, 80 (4), 1505-1531.

[Reynaud and Couture]Reynaud and Couture2012Reynaud/Couture:2012 Reynaud, Arnaud and Stéphane Couture, "Stability of risk preference measures: results from a field experiment on French farmers," Theory and Decision, 2012, 73 (2), 203-221.

[Roberts et al.]Roberts, Kuncel, Shiner, Caspi and Goldberg2007Roberts/Kuncel/Shiner:2007 Roberts, Brent W, Nathan R Kuncel, Rebecca Shiner, Avshalom Caspi, and Lewis R Goldberg, "The power of personality: The comparative validity of personality traits, socioeconomic status, and cognitive ability for predicting important life outcomes," Perspectives on Psychological Science, 2007, 2 (4), 313-345.

[Rustichini et al.]Rustichini, DeYoung, Anderson and Burks2012RustichiniEtAl:2012 Rustichini, Aldo, Colin G DeYoung, Jon C Anderson, and Stephen V Burks, "Toward the 
integration of personality theory and decision theory in the explanation of economic and health behavior," 2012. IZA Discussion Paper.

[Scheinkman and Xiong]Scheinkman and Xiong2003Scheinkman/Xiong:2003 Scheinkman, Jose A and Wei Xiong, "Overconfidence and speculative bubbles," Journal of Political Economy, 2003, 111 (6), 1183-1220.

[Strotz]Strotz1955Strotz:1955 Strotz, R. H., "Myopia and Inconsistency in Dynamic Utility Maximization," The Review of Economic Studies, 1955, 23 (3), pp. 165-180.

[Tversky and Kahneman]Tversky and Kahneman1991Tversky/Kahneman:1991 Tversky, Amos and Daniel Kahneman, "Loss aversion in riskless choice: A reference-dependent model," The Quarterly Journal of Economics, 1991, 106 (4), 1039-1061.

[van Witteloostuijn and Muehlfeld]van Witteloostuijn and Muehlfeld2007vanWitteloostuijnEtal:2007 van Witteloostuijn, Arjen and Katrin Muehlfeld, "Trader personality and trading performance," 2007. Tjalling C. Koopmans Research Institute Discussion Paper Series \# nr: 08-28. 